\title{
The Condominium Problem; Auctions for Substitutes
}

\author{
Roberto Burguet \\ Institute for Economic Analysis (CSIC)
}

This version: February 2002

\begin{abstract}
This paper considers the problem of designing selling procedures for substitutes (like condominium units). I show that oral, ascending auctions for the right to choose are efficient. This is a common type of auction used for the sale of real estate. Efficiency is not optimal from the seller's viewpoint. An optimal procedure distorts the right-tochoose auction to favor in late rounds bidders whose preferred object has already been sold. This optimal auction is complex. A revenue improving departure from efficiency can be achieved by simply auctioning all the rights to choose before any of them is exerted. This is also a common feature of auctions for the sale of real state.

JEL Classification: D44.

Keywords: Auctions, Right-to-choose

Address: Institute for Economic Analysis (CSIC)

Campus de la UAB

08193-Bellaterra (Barcelona)

Spain

e-mail: roberto.burguet@uab.es

I acknowledge very helpful comments by Joe Harrington, Ken Hendricks, and two anonymous referees. Financial aid from the European Commission through the TMR Program (contract FMRX-CT98-0203) is also gratefully acknowledged.
\end{abstract}




\section{Introduction}

Put yourself in the shoes of the owner of a number of apartments in some apartment building, who is considering selling these apartments. How would you proceed? Faced with this question, your first move would probably be to turn to auction theory for help. ${ }^{1}$ There you would quickly find advice on how to sell several units of homogeneous goods to buyers who are willing to acquire at most one unit. You may even find some results that apply when buyers are willing to buy more than one unit. Yet, you may wonder how much these results teach you about your problem. After all, your apartments are not "exactly" homogeneous. They may be "substitutes" but they are not "perfect substitutes". Some face west, some face east. Some receive more sunshine but are noisier too. In fact, no two apartments are identical. Not only they are different, but you may find it difficult to decide which one is "better". Different buyers may be expected to give you different answers to that question. If you keep looking for advise at auction theory, you may find some discussions on the sale of distinct (yet related) objects. But soon you will realize that in these discussions, for some unspecified reason, buyers are assumed to leave the auction once they own one object, irrespective of the price they paid or the object they get. ${ }^{2}$

Other than that, there will be little guidance for your problem, which I term "the condominium problem". That is, the sale of several objects with the following features:

-Buyers have different willingness to pay for different objects: objects are distinct.

-The willingness to pay for one of the objects depends on whether the buyer already owns another one. In particular, owning one object reduces the willingness to pay for another one, perhaps to zero.

\footnotetext{
${ }^{1}$ A couple of recent surveys to look at would be Klemperer (1999) or Burguet (2000).

${ }^{2}$ This is what Bernhardt and Scoones (1994), and Gale and Hausch (1994) assume.
} 
Buyers who like an apartment facing west more than an apartment facing east would be willing to pay more for the former than for the latter. On the other hand, if buyers' main goal is to buy a place to live in, once they get one apartment, say one facing east, they would be willing to pay less for another one facing west, although they would still be willing to pay a positive amount: at least their money value for having their more preferred, west-facing, apartment.

The goal of this paper is to investigate this problem, which, to the best of my knowledge, has so far suffered from neglect. ${ }^{3}$ First I study what allocation of objects to buyers would be efficient in this set up. That is, how the objects should be assigned to buyers in order to maximize the gains from trade. Then I show that this efficient allocation can be implemented with oral, ascending auctions for the right to choose, also known as "pooled" auctions, which in fact are commonly used to sell condominium units (see Ashenfelter and Genesove (1992)). These are sequential, English auctions in which the winner of a round wins not a specific object, but the right to choose any of the yet unsold objects. In fact, these are auctions often. Efficiency requires that winners of early rounds make their choices before the beginning of later rounds.

Next I study the problem from the point of view of the seller. That is, I investigate what type of selling procedure (auction) maximizes the seller's expected revenues (the optimal auction). In standard auction theory, efficiency and optimality are very closely related. For instance, under symmetry and private values, and if one does not consider the possibility of leaving the object unsold, any auction that assigns the good efficiently is also optimal for the seller of a single good. In our condominium problem this is not true. Even if buyers are

\footnotetext{
${ }^{3}$ Due to the boom in auctions of spectrum licencies, a lot of attention has been devoted to the sale of this type of commodities. It is usually considered that willingness to pay for a licence (when owning more than one is allowed, as in some of the FCC auctions) is affected by the possesion of another licence. However, in that case the main link seems to come from complementarities rather than substituibilities.
} 
symmetric ex-ante, once their most preferred object has already been taken, bidders become weaker competitors vis-à-vis buyers whose most preferred objects are yet unsold. Thus, in a way completely in line with what we know about asymmetric auctions, and incentive problems in general, distorting the allocation to favor these bidders helps reducing the informational rents that other, stronger bidders obtain.

These "optimal distortions" may have little practical appeal. As in the asymmetric buyers case in auctions for a single object, they require complex auction rules, and a detailed knowledge of the distribution of buyers' preferences. From a more practical point of view, however, there are simple designs that induce distortions in this direction and consequently increase the seller's revenues. Indeed, I show that the seller can increase her revenues by auctioning all the rights to choose before any one is exerted, as is often done in real estate auctions.

As an incidental result, this paper also explains the tendency of prices in successive rounds to decline, as observed for these same auctions by Ashenfelter and Genesove (1992). By now we are used to find that the once termed "declining price anomaly" is not so much of an anomaly. Yet, in this paper I show that in right-to-choose auctions rather than anomaly declining prices should be considered as the rule. After all, the value of what is offered for sale, the right to choose among the shrinking set of objects not sold before, declines too.

In the next section I present the model and main assumptions. Section 3 studies efficiency and shows that right-to-choose auctions implement the efficient allocation. Section 4 is devoted to revenue equivalence and revenue maximization. Then Section 5 concludes with a discussion of price patterns and extensions.

\section{The model}

There are $n$ potential buyers for two distinct objects, $E$ and $W$ (East and West). Buyer i's preferences, $i=1,2, \ldots, n$ are characterized by two parameters $\left(v_{i}, \theta_{i}\right)$, 
where $\theta_{i} \in\{E, W\}$, is the "location type", and $v_{i} \in[0,1]$, is the "valuation type". For each $i, \theta_{i}$ takes the value $E$ with a probability of $\frac{1}{2}$ and the value $W$ with a probability of $\frac{1}{2}$. Also, $v_{i}$ is the realization of a random variable with c.d.f. $F$ and density $f$ which takes positive values on $[0,1]$. All random variables are independent. All this is common knowledge. In addition, buyer $i$ knows the realization of $\left(\theta_{i}, v_{i}\right)$.

The location type of a buyer determines her most preferred object. The valuation type determines how much the buyer is willing to pay for this each object. We also assume away income effects in the preferences of buyers. Thus, the pay-off for buyer $i$ with parameters $\left(\theta_{i}, v_{i}\right)$ who obtains object $I=\theta_{i}$, or both objects at a price $P$ is

$$
U\left[I=\theta_{i}, P ;\left(\theta_{i}, v_{i}\right)\right]=v_{i}-P .
$$

The pay-off for this buyer if she obtains her less preferred object $I \neq \theta_{i}$ (and only this object) is

$$
U\left[I \neq \theta_{i}, P ;\left(\theta_{i}, v_{i}\right)\right]=l\left(v_{i}\right)-P,
$$

where $l$ is a strictly monotone increasing function. We assume $l(0)=0$. Also, $1>l^{\prime}(v)$. For instance, the willingness to pay for the less preferred object may be a fixed proportion of the willingness to pay for the most preferred. ${ }^{4} 5$ Finally, the pay-off for buyer $i$ who pays $P$ and gets no object is $-P$.

We are portraying a situation in which, although the two objects can be identified (they are distinct, as two apartments in the same condominium would be), they are symmetric from an ex-ante point of view. That is, neither of them

\footnotetext{
${ }^{4}$ Notice that, as opposed to what Gale and Hausch (1994) (implicitly) assume, a buyer is willing to pay a positive amount to get her most preferred object even if she already owns her less preferred one.

${ }^{5}$ Later we will discuss the extension of this "unit demand" model for a case where the buyer is willing to pay a positive amount for the less preferred object even when she already owns her most preferred one.
} 
is ex-ante more valuable than the other. Also, notice that buyers are assumed risk neutral. Finally, for simplicity we assume that the seller's valuation for both objects is sufficiently small (non-positive). We next turn to discussing efficiency and its implementation in this setting.

\section{Efficiency: oral, ascending auctions for the right-to-choose}

Efficiency under these assumptions is easy to characterize. Without loss of generality, assume buyer 1 has the highest valuation type, buyer 2 has the second highest, etc. Then, if $\theta_{1} \neq \theta_{2}$ (buyers 1 and 2 prefer different objects), buyers 1 and 2 should receive their most preferred objects. If $\theta_{1}=\theta_{2}$ then we may have to consider a third buyer: the one with highest valuation type among those with location type other than $\theta_{1}$. Call this buyer buyer $d i$ (for different). Then again buyer 1 should get her most preferred object, but who gets the other depends now on which of the values: $l\left(v_{2}\right)$, or $v_{d i}$ is highest. It should go to buyer 2 in the first case, and to buyer $d i$ in the second.

Consider now selling the objects using an oral, ascending auction for the right to choose. As usual, we model English auctions by assuming that a clock points to continuously ascending prices, and buyers keep their hands up for as long as they wish. The round ends once all but one of them lower their hands. The winner is the buyer left. The price is the one the clock shows at the moment the penultimate buyer dropped her hand.

Oral ascending auctions for the right to choose is a common mechanism used in sales of condominium units. It consists of a sequence of regular English auctions in which the winner does not get any prespecified object, but the right to choose any one among the yet unsold objects. In our case, we would have only two rounds. It is not difficult to see that the winner of the first would certainly choose her most 
preferred object. Also, if every bidder observes the choice of this winner, in the second (last) auction every buyer's best strategy is to bid up to her willingness to pay for the remaining object. Thus, if buyers' equilibrium strategy in the first auction is such that the winner is the buyer with highest $v_{i}$, independently of $\theta_{i}$, then this mechanism indeed implements an efficient allocation. The first proposition simply states that this is the case.

Proposition 1 The oral, ascending, aution for the right to choose has an efficient equilibrium.

Proof. See Appendix.

The equilibrium strategies are cumbersome to write but simple to interpret. As usual, when dropping at a price of $P$ a buyer should weigh the gains and losses of staying a little longer, and should find them equal. Dropping and staying a little longer result in different outcomes for the buyer only if all remaining buyers are considering dropping at (approximately) that same price. In a monotone, continuous equilibrium, ${ }^{6}$ this happens when all remaining buyers have the same valuation type (but perhaps not the same location type) as the buyer considered. On the other hand, again under monotonicity, the dropping prices of buyers who have already dropped reveal their valuation type. Then, these are the conjectures under which the equilibrium dropping price is computed by all buyers. The only novelty in our set-up is that valuation types $v_{i}$ do not determine the other type dimension: location type $\theta_{i}$. So what happens in the second period is not completely deterministic conditioning on the observations and conjectures made in the first round, but some randomness remains.

\footnotetext{
${ }^{6}$ Strategies are described by vectors of functions, one for each number of bidders that have already dropped. Each function gives the dropping price for the buyer under the assumption that no other drop occurs, and given the valuations of drop-outs inferred from their dropping price and the valuation type of the buyer. Monotonicity here means that all those functions are monotone. Continuity means also that when one more drop occurs the new dropping price for a buyer that was indifferent between dropping and staying is unchanged.
} 
We are able to show that strategies computed in this way are monotone, continuous, and are indeed equilibrium strategies. Then the efficiency of rightto-choose auctions follows.

As an illustration, consider the following three buyer example, $n=3$. Assume no buyer has yet dropped. Then, the dropping price $P_{0}\left(v_{i}\right)$ for a buyer $i$ with valuation $v_{i}$ (and any $\theta_{i}$ ) equates what the buyer gets if dropping now under the conjecture that the rest of the buyers have valuations $v_{i}$,

$$
\left(\frac{1}{2}\right)^{2}\left[v_{i}-l\left(v_{i}\right)\right]
$$

with what the buyer expects if staying under that same assumption

$$
\left[v_{i}-P_{0}\left(v_{i}\right)\right]
$$

Indeed, dropping means that the buyer gets positive surplus only if the other buyers have a location type different than her own type. In this event, which has probability $\frac{1}{4}$, one of the other bidders (the loser) will bid $l\left(v_{i}\right)$ in the second round. In any other case, either the buyer gets no object in the second round or she has to pay a price equal to her own willingness to pay for the object left. On the other hand, if staying the buyer gets to choose her most preferred object. Thus, the dropping price in the first round is defined by

$$
P_{0}\left(v_{i}\right)=\frac{3 v_{i}+l\left(v_{i}\right)}{4}
$$

This is an increasing function of $v_{i}$. Similarly, one can compute the dropping price $P_{1}\left(v_{i} ; v_{3}\right)$ for a buyer with valuation type $v_{i}$ when one other bidder has already dropped at a price that reveals (according to the above equation) a valuation of $v_{3}$. Assume, for instance, that $v_{3}>l\left(v_{i}\right)>l\left(v_{3}\right)$. That is, we were already approaching the dropping decision of buyer $i$ when buyer 3 dropped. Then, by dropping under the assumption that the other remaining buyer has also valuation $v_{i}$, buyer $i$ will obtain the second object only if this remaining buyer has different 
location type, or if she has the same location type but buyer 3 also has the same location type. This means expected surplus of ${ }^{7}$

$$
\left(\frac{1}{2}\right)\left(v_{i}-\frac{1}{2} l\left(v_{3}\right)-\frac{1}{2} v_{3}\right)+\left(\frac{1}{2}\right)^{2}\left(l\left(v_{i}\right)-l\left(v_{3}\right)\right) .
$$

By staying longer under the same conjectures she is certain to get her most preferred object. Thus,

$$
P_{1}\left(v_{i} ; v_{3}\right)=\frac{2 v_{i}+v_{3}+2 l\left(v_{3}\right)-l\left(v_{i}\right)}{4} .
$$

This is also an increasing function of $v_{i}$. Moreover, when $v_{i}=v_{3}, P_{1}\left(v_{i} ; v_{3}\right)=$ $P_{0}\left(v_{i}\right)$. That is, when one buyer drops (buyer 3 ), a buyer with strictly higher valuation will still wait a little before dropping. This continuity guarantees that the two remaining bidders do not drop at the same time, and then guarantees that the buyer with highest valuation wins the first round.

\section{Maximizing the seller's revenue}

We now investigate how a seller should design her selling mechanism in order to maximize her expected revenue. This section generalizes well known results on revenue equivalence and the allocation properties of optimal auctions (see Myerson, 1981) to encompass the sale of substitutes. For any mechanism, denote by $p_{i}^{I}(\mathbf{v} ; \boldsymbol{\theta})$ the probability that buyer $i$ obtains object $I$ when valuation types are $\boldsymbol{\theta}=\left(\theta_{1}, \theta_{2}, \ldots, \theta_{n}\right)$ and location types are $\mathbf{v}=\left(v_{1}, v_{2}, \ldots, v_{n}\right)$. Also, denote by $\Omega_{-i}$ the type space for $n-1$ buyers, $\Omega_{-i}=([0,1] \times\{E, W\})^{n-1}$, and by $\mu$ the probability measure induced on this set by the random variables $v_{j}, \theta_{i}, j \neq i$. Also, let

$$
\rho_{i}^{E}\left(v_{i} ; \theta_{i}\right)=\int_{\Omega_{-i}} p_{i}^{E}\left((\mathbf{v} ; \boldsymbol{\theta})_{-i} ;\left(v_{i}, \theta_{i}\right)\right)\left[1-p_{i}^{W}\left((\mathbf{v} ; \boldsymbol{\theta})_{-i} ;\left(v_{i}, \theta_{i}\right)\right)\right] d \mu\left(\Omega_{-i}\right),
$$

\footnotetext{
${ }^{7}$ If $l\left(v_{i}\right)>v_{3}$, we should add to this $\left(\frac{1}{4}\right)\left(l\left(v_{i}\right)-v_{3}\right)$, and change the expression for $P_{1}$ accordingly.
} 
that is, the expected probability that buyer $i$ obtains object $E$, but only $E$, given that her location type is $\theta_{i}$ and her valuation type is $v_{i}$. Define similarly $\rho_{i}^{W}\left(v_{i} ; \theta_{i}\right)$. Finally, let

$$
\rho_{i}^{B}\left(v_{i} ; \theta_{i}\right)=\int_{\Omega_{-i}} p_{i}^{E}\left((\mathbf{v} ; \boldsymbol{\theta})_{-i} ;\left(v_{i}, \theta_{i}\right)\right) p_{i}^{W}\left((\mathbf{v} ; \boldsymbol{\theta})_{-i} ;\left(v_{i}, \theta_{i}\right)\right) d \mu\left(\Omega_{-i}\right)
$$

be the probability that buyer $i$ obtains both objects. The following lemma should not be surprising.

Lemma: (Revenue equivalence) Two mechanisms with common $\rho_{i}^{I}, I=$ $E, W$, and $\rho_{i}^{B}$ are revenue and rent equivalent if each buyer $i$ with type $\left(0, \theta_{i}\right)$, $\theta_{i}=E, W$ obtains the same rents in both. Buyer $i$ 's expected rents when $\theta_{i}=E$ are given by

$$
\pi_{i}\left(v_{i} ; E\right)=\int_{0}^{v_{i}}\left[\rho_{i}^{E}(z ; E)+\rho_{i}^{B}(z ; E)+l^{\prime}(z) \rho_{i}^{W}(z ; E)\right] d z+\pi_{i}(0 ; E),
$$

and similarly when $\theta_{i}=W$.

Proof. See Appendix.

Consider now the problem of maximizing the seller's expected revenue in the set of all possible auctions. As usual, notice that this is equivalent to maximizing the difference between total surplus (gains from trade) and expected buyers' rents. Define the function $J: R \rightarrow R$, the "virtual valuation" (see, for instance, Myerson, 1981), as

$$
J(v)=v-\frac{1-F(v)}{f(v)}
$$

and define $J_{l}: R \rightarrow R$ as

$$
J_{l}(v)=l(v)-\frac{1-F(v)}{f(v)} l^{\prime}(v) .
$$

We make the traditional regularity assumption, that $J\left(v_{i}\right)$ is increasing in $v_{i}$, but also that $J_{l}\left(v_{i}\right)$ is increasing in $v_{i} \cdot{ }^{8}$ As we will see, here efficiency will not be synonym of optimality even under this assumption. However, with our monotonicity assumptions, we can characterize the type of distortions that maximize

\footnotetext{
${ }^{8}$ Monotonicity of the inverse hazard rate $\frac{1-F(v)}{f(v)}$ and concavity of $l$ already imply this.
} 
the seller's revenue. We restrict attention to auctions that assign the objects to buyers with probability 1 , so that we do not have to consider the reservation value of the seller or the commitment not to sell. The extension to include the possibility of not selling is straightforward. Thus, the following proposition offers the characterization of optimal auctions. We still use the convention that $v_{1}$ is the highest realization of the valuation type, $v_{2}$ the second largest, etc. Also, again let $d i$ be the buyer with highest valuation type among those with location type other than $\theta_{1}$.

Proposition 2 An optimal auction assigns buyer 1 her most preferred object. If $\theta_{1} \neq \theta_{2}$, then the optimal auction assigns the second object to buyer 2 . If $\theta_{1}=\theta_{2}$, then the optimal auction assigns the second object to buyer 2 or buyer di, respectively if $J_{l}\left(v_{2}\right)$, or $J\left(v_{d i}\right)$ (if di exists) is higher.

Proof. See Appendix.

As opposed to what happens in the standard auction case, this proposition means that optimal (from the seller's viewpoint) auctions for substitutes will be inefficient. In particular, under our assumptions,

Corollary 2: The optimal auction allocates the two objects to buyers with the same location type more often than efficient.

Indeed, if $l\left(v_{2}\right)=v_{d i}$, then $J_{l}\left(v_{2}\right)>J\left(v_{d i}\right)$. This distortion should sound familiar to the reader familiar with auction theory: in the second auction, a buyer with location type equal to $\theta_{1}$ is "weaker" in that she obtains her willingness to pay (for the second object) from a less favorable distribution.

For the sake of illustration, consider the case where $v_{i}$ is distributed uniformly and $l$ is linear: $l\left(v_{i}\right)=l v_{i}$. To simplify further, with no further loss of generality, assume $n=3$. In this case $J\left(v_{i}\right)=2 v_{i}-1$ and $J_{l}\left(v_{i}\right)=l J\left(v_{i}\right)$. Consider the following modifications to the oral, ascending action for the right to choose:

-The last buyer to drop (who sets the price in the first round) has to pay a 
fee that depends on the dropping price of buyer 3 ,

$$
\phi\left(v_{3}\right)=\left\{\begin{array}{cl}
\frac{1-l}{2} & \text { if } v_{3} \leq \frac{1}{2} \\
\frac{1-l}{2}-\left(v_{3}-\frac{1}{2}\right) \frac{3(1-l)}{4} & \text { if } v_{3}>\frac{1}{2}
\end{array},\right.
$$

in order to participate in the second round. (The fee is equal to the expected payoff if buyer 2 has valuation type $v_{3}$ too.)

-The first buyer to drop is handicapped by $\frac{1-l}{2}$ in the second round. That is, she loses that round unless she beats the other buyer (buyer 2) by at least that amount.

Using the same notation of the previous section, then equilibrium behavior means that buyers drop at prices

$$
P_{0}\left(v_{i}\right)=\left\{\begin{array}{cl}
v_{i} & \text { if } v_{i} \leq 1 / 2 \\
l v_{i}+\frac{1-l}{2} & \text { if } v_{i}>1 / 2
\end{array},\right.
$$

and

$$
P_{1}\left(v_{i} ; v_{3}\right)=\left\{\begin{array}{cc}
\frac{v_{i}+v_{3}-l\left(v_{i}-v_{3}\right)}{2} & \text { if } v_{3} \leq 1 / 2 \\
\frac{v_{i}+v_{3}-l\left(v_{i}-v_{3}\right)}{2}-\frac{3}{4}(1-l)\left(v_{3}-\frac{1}{2}\right) & \text { if } v_{3}>1 / 2 \\
\frac{v_{i}+v_{3}-\frac{1}{2} l\left(v_{i}-v_{3}\right)}{2}-(1-l)\left(v_{3}-\frac{1}{2}\right) & \text { ind } l v_{i}+\frac{1-l}{2} \geq v_{3}>1 / 2 \\
& \text { and } l v_{i}+\frac{1-l}{2}<v_{3}
\end{array} .\right.
$$

Again, we can check both continuity and monotonicity of these two functions, so that $v_{3}$ is trivially inferred from $P_{0}$, and also $P_{0}\left(v_{i}\right)=P_{1}\left(v_{i} ; v_{i}\right)$, so that the first round is won by the highest valuation buyer. It is also straightforward to check that this is an optimal auction for this particular case.

As in the problem of designing optimal auctions for a single object when buyers are asymmetric (see, for instance, Maskin and Riley (1984)), the type of favoritism that buyers should enjoy depends on the types of the buyers (perhaps inferred from their bid behavior). Also, detailed information about the distribution of this types is needed. This together with the complexity of the 
optimal mechanism in general explains why we hardly observe auctions like the one outlined above.

However, an important, practical question is whether other simple auctions may improve upon the unmodified oral, ascending auction for the right to choose (i.e., upon an efficient auction). ${ }^{9}$ We show next that indeed there is a simple modification to our right to choose auction that attains this goal, one that in fact is commonly observed in auctions of real estate. This modification consists of simply auctioning all rights to choose before any one is exerted. That is, in our two object case, the choice of the winner in the first round would not yet be known when bidders bid in the second round. It is straightforward to show that the winner in round $n=1,2$ would be $i=n$. That is, buyer 2 would win the second object even if buyer 3 is willing to pay more for this object (because $\theta_{1}=\theta_{2} \neq \theta_{3}$, and $\left.l\left(v_{2}\right)<v_{3}\right)$. This type of inefficiency is indeed in line with the ones that an optimal mechanisms would incorporate, and in fact,

Proposition 3 In an auction for the right to choose, keeping secret buyer 1's choice increases the expected revenues for the seller.

Proof. See Appendix.

\section{Discussion}

\subsection{Declining prices}

A tendency for the sequence of prices of repeated sales to decline has been widely observed. In particular, this has been observed by Ashenfelter and Genesove (1992) in the auctioning of condominium units when sellers use right-to-choose

\footnotetext{
${ }^{9}$ Standard sequential auctions, where objects, and not the right to choose, are auctioned in sequence, are not good alternatives. Indeed, coordination problems should render this auction format inefficient (high valuation bidders may obtain both objects, if rival bidders have sufficiently low valuation, for instance), but in ways that do not help raising the seller's revenue.
} 
auctions. The authors are quick to point out that such price behavior should be expected if the units are not homogeneous, so that early winners can buy superior units. However heterogeneity of the units does not entirely explain the downward drift, according to the authors. Here, we have been considering a case of "symmetric" (from an ex-ante point of view, of course) objects. Let us return to our three buyer case in Section 3, with observed choices of previous winners (the exercise without observability is even simpler). ${ }^{10}$ Then, given $v_{3}$ and $v_{2}$ we can compute the price in the first round and the expected price in the second. In the first round the price is given by $P_{1}\left(v_{2} ; v_{3}\right)=\frac{2 v_{2}+v_{3}+2 l\left(v_{3}\right)-l\left(v_{2}\right)}{4}$ if $v_{3}>l\left(v_{2}\right)$, and $\frac{v_{2}+v_{3}-\left(l\left(v_{2}\right)-l\left(v_{3}\right)\right)}{2}$ otherwise. In the second round, the price will be $l\left(v_{3}\right)$ if both remaining buyers have the same location type as the winner of the first round, or if buyer 3 only has the same location type as the winner. It will be equal to $v_{3}$ if both have location types different from the winner of the first round. The price will be $\min \left\{l\left(v_{2}\right), v_{3}\right\}$, otherwise. Each of the events above has a probability of $1 / 4$. Thus, the price expected for the second round will be

$$
\frac{2 l\left(v_{3}\right)+v_{3}+\min \left\{l\left(v_{2}\right), v_{3}\right\}}{4} .
$$

Thus, the price in the first round is $\frac{v_{2}-l\left(v_{2}\right)}{2}$ higher than the price in the second round. This difference is easy to interpret. It is exactly the value of choosing in the first place instead of the second (which is set by buyer 2). Even though the two objects are stochastically equivalent in terms of value from an ex-ante point of view, the ability to choose has a price. When both objects are indistinguishable $(l(v)=v)$, this difference is nil, and we recover the martingale property of prices in sequential auctions.

\footnotetext{
${ }^{10}$ With multiunit demands, prices should also be expected to decline. See, for instance, Black and de Meza (1992), Burguet and Sákovics (1997), or Bernhardt and Scoones (1994).
} 


\subsection{The value of diversity}

When $l(v)=v$, the model we have been considering reduces to the standard two unit auction with unit demand bidders. Taking this as a benchmark, the case where $l(v)<v$ can be seen as a reduction of value of the objects for sale. Indeed, buyers' willingness to pay for the objects, on average, is lower. It is natural to expect that using optimal mechanisms, the revenues that a seller can obtain in this second case should then be lower. In fact, they need not be.

Consider for instance the case $n=3$. If buyers are indifferent among the objects, then the expected revenue of the revenue maximizing auction is simply twice the expected value of the third order statistic. That is, the expected value of $2 v_{3}$. Indeed, under the assumption that the objects are sold with probability 1 , any efficient mechanism is efficient and produces that revenue. However, if buyers value one of the objects at $l(v)$, as in our case, our right to choose auction without observability of choices (which is not even optimal) extracts revenue equal to $v_{3}+l\left(v_{3}\right)+\frac{v_{2}-l\left(v_{2}\right)}{2}$. This is $\frac{v_{2}-l\left(v_{2}\right)}{2}-\left(v_{3}-l\left(v_{3}\right)\right)$ in excess of $2 v_{3}$. This difference may well be positive. For instance, if $v$ 's are uniform and $l(v)=v\left(1-\frac{v}{10}\right)$, this difference is .005 .

\section{Concluding remarks}

We have investigated the implementation of efficient and optimal allocation for the condominium problem. That is, for the sale of substitutes. Right to choose auctions, which are commonly used for this type of sales, are efficient if choices are exerted right after the allocation of such right. However, keeping this choices until all the rights have been auctioned increases the seller's revenues. We have assumed throughout that buyers have unit demands. Indeed, buyers were willing to pay a positive amount to "upgrade" their unit . That is, to buy the most preferred object even when they already owned the other object. But buyers 
were indifferent between owning two objects and owning their most preferred one. Most of the results above would extend easily to a scenario where buyers are also willing to pay a positive amount for the less preferred object when they already own their favorite. Assume that a buyer with valuation $v_{i}$ is willing to pay $b\left(v_{i}\right)>v$, with $l^{\prime}>b^{\prime}-1>0$. One can easily show that the auction for the right to choose where choices are announced before subsequent rounds also implements the efficient allocation. This allocation would require comparing $b\left(v_{1}\right)$ with $v_{2}$, or $l\left(v_{2}\right)$ and $v_{d i}$ (depending on whether $\theta_{2}$ equals $\theta_{1}$ or not) to decide who obtains the second object.

Revenue equivalence also holds under this assumption. The optimal mechanism for this case adds a new type of inefficiency: excessive bundling. That is, buyer 1 obtains the two objects even when buyer 2 is willing to pay more for the second object. With multiple unit demand, keeping choices secret, however does not unambiguously increase the seller's revenues.

The one dimensionality of signals was certainly a strong assumption. Indeed, if preferences were multidimensional (if buyers with the same willingness to pay for one object had different willingness to pay for the other) the right to choose auction could not possibly be efficient. In fact, no simple mechanism would. Thus, the results in this paper should be understood as approximate, but not exact, when the willingness to pay for the two objects are correlated, but not perfectly correlated.

\section{References}

[1] Ashenfelter, O. and D. Genesove (1992) "Testing for Price Anomalies in Real-Estate Auctions" American Economic Review, 82-2, pp. 501-505

[2] Bernhardt, D. and D. Scoones "A Note on Sequential Auctions", American Economic Review, 84, pp.653-657 
[3] Black J. and D. de Meza (1992) "Systematic Price Differences Between Successive Auctions Are no Anomaly", Journal of Economics and Management Strategy 1, pp. 60\%-628.

[4] Burguet, R. (2000) "Auction Theory; A Guided Tour", Investigaciones Económicas, 34, pp. 3-50

[5] Burguet R., and J. Sákovics (1997) "Sequential Auctions with Demand or Supply Uncertainty" Revista Española de Economía 14, pp. 23-40.

[6] Gale, I. and D. Hausch (1994) "Bottom-Fishing and Declining Prices in Sequential Auctions" Games and Economic Behavior 7, 318-331

[7] Klemperer, P. (1999) "Auction Theory; A Guide to the Literature", Journal of Economic Surveys, 13, pp. 227-286

[8] Maskin, E. and J. Riley (1985) "Auction Theory with Private Values" American Economic Review, 75, pp. 150-155

[9] McAfee, P. and J. McMillan (1987) "Auctions with Entry", Economics Letters, 23, pp. 343-347.

[10] Myerson, R. (1981) "Optimal Auction Design", Mathematics of Operations Research 6, pp. 58-73 


\section{A Appendix}

Proof of Proposition 1:The second round is trivial from the strategic point of view: buyer's dominant strategy is to bid up to willingness to pay for whichever object is left. For the first round, we define the equilibrium strategies inductively. Assume $j<n-2$ buyers have dropped revealing valuation types $\left(v_{n}, v_{n-1}, \ldots, v_{n-j+1}\right)$. This includes trivially the case that no buyer has dropped yet. We will see that indeed valuation types can be inferred from dropping prices. Let $k, n \geq k \geq n-j$ be such that $v_{k} \geq l\left(v_{i}\right) \geq v_{k}+1$. That is, $k$ is the highest index of the buyer among the ones that have already dropped and whose willingness to pay for her most preferred object exceeds buyer $i$ 's willingness to pay for her less preferred object. When $l\left(v_{i}\right)$ is higher than $v_{m}$ for $m=n, n-1, \ldots, n-j+1$, then $k=n-j$. We compute the dropping price for a buyer with valuation type $v_{i}$ similarly to the way we did for the case $n=3$. That is, we assume that the buyer conjectures that all other buyers that remain in the auction have valuation $v_{i}$ too. Under this conjecture we compute how much the buyer expects if dropping at the current price and how much she gets by staying. By staying the buyer wins the first round at the going price. So we also have to check that indeed under the conjectures of the buyer, all other buyers' equilibrium behavior is to drop at the current price. Thus:

-Staying: the price will be the current price $P$, the buyer chooses her most preferred object. Thus, staying means a pay-off of $v_{i}-P$.

-Dropping: one buyer will get her most preferred object, which may be buyer $i$ 's most preferred object or not, each event with probability $1 / 2$. Then other $n-j-2$ buyers have valuation type $v_{i}$. When $j<n-2$, buyer $i$ will not get a positive payoff (either because the price in the second round will reach her willingness to pay or because she will be defeated by other bidders) unless the winner and the other $n-j-2$ buyers with valuation $v_{i}$ have different location type from that of buyer $i$. In this latter case, buyer $i$ wins the second (her most 
preferred) object, and the price will go up to $l\left(v_{i}\right), v_{k}, v_{k-1}, \ldots$ or $v_{n-j+1}$, depending on whether all buyers $k, \ldots, n-j+1$ have also the same location type of the winner, or all $k-1, \ldots, n-j+1$ have that location type but not buyer $k$, etc. Thus, for $j<n-2$, the pay-off expected if dropping is

$$
\begin{aligned}
\left(\frac{1}{2}\right)^{n-j-1} & \left\{\sum_{m=1}^{k-(n-j)}\left(\frac{1}{2}\right)^{m}\left[v_{i}-v_{n-j+m}\right]+\left(\frac{1}{2}\right)^{k-(n-j)}\left[v_{i}-l\left(v_{i}\right)\right]\right\}= \\
& \left(\frac{1}{2}\right)^{n-j-1} v_{i}-\left\{\sum_{m=n-j+1}^{k}\left(\frac{1}{2}\right)^{m-1} v_{m}+\left(\frac{1}{2}\right)^{k-1} l\left(v_{i}\right)\right\} .
\end{aligned}
$$

Thus, for $j<n-2$, we propose

$$
\begin{aligned}
& P_{j}\left(v_{i} ; v_{n}, v_{n-1}, \ldots, v_{n-j+1}\right)= \\
& v_{i}\left(1-\left(\frac{1}{2}\right)^{n-j-1}\right)+\left\{\sum_{m=n-j+1}^{k}\left(\frac{1}{2}\right)^{m-1} v_{m}+\left(\frac{1}{2}\right)^{k-1} l\left(v_{i}\right)\right\} .
\end{aligned}
$$

When $j=n-2$, that is, when only one other bidder is left, things are slightly different. In particular, buyer $i$ could obtain a positive pay-off even when her location type coincides with that of buyer 1 . Thus, let $k^{\prime}(\geq k)$ be the integer that satisfies $v_{k^{\prime}} \geq l\left(v_{3}\right) \geq v_{k^{\prime}}+1$. The pay-off when dropping in this case is

$$
\frac{1}{2} v_{i}+\left(\frac{1}{2}\right)^{k-1} l\left(v_{i}\right)-\left\{\sum_{m=3}^{k}\left(\frac{1}{2}\right)^{m-1} v_{m}+\sum_{m=k+1}^{k^{\prime}}\left(\frac{1}{2}\right)^{m-2} v_{m}+\left(\frac{1}{2}\right)^{k^{\prime}-2} l\left(v_{3}\right)\right\},
$$

and then,

$$
\begin{aligned}
& P_{2}\left(v_{i} ; v_{n}, v_{n-1}, \ldots, v_{3}\right)= \\
& \frac{1}{2} v_{i}-\left(\frac{1}{2}\right)^{k-1} l\left(v_{i}\right)+\left\{\sum_{m=3}^{k}\left(\frac{1}{2}\right)^{m-1} v_{m}+\sum_{m=k+1}^{k^{\prime}}\left(\frac{1}{2}\right)^{m-2} v_{m}+\left(\frac{1}{2}\right)^{k^{\prime}-2} l\left(v_{3}\right)\right\} .
\end{aligned}
$$

These are all monotone functions of $v_{i}$. Also, $P_{j}\left(v_{i} ; v_{n}, v_{n-1}, \ldots, v_{n-j+1}\right)=P_{j+1}\left(v_{i} ; v_{n}, v_{n-1}, \ldots, v_{n-j+1}\right.$, $\left.v_{i}\right)$. That is, if a drop $j+1$ happens exactly when buyer $i$ was planning to drop, buyer $i$, but not any buyer with valuation higher than hers, will also drop. These were the two conjectures that we had to check. From this too, optimality of behavior follows trivially: dropping before the correponding price only makes a 
difference when the expected pay-off in the first round is higher, and dropping after that price only makes a difference when the expected pay-off in the second round is higher.QED.

Proof of Lemma (Revenue equivalence):

The proof is very standard: Let $X_{i}\left(v_{i} ; \theta_{i}\right)$ denote the expected payments of buyer $i$ with valuation type $v_{i}$ and location type $\theta_{i}$. For a location type $E$, for instance, incentive compatibility requires (among other things) that

$$
v_{i}=\arg \max _{z}\left[\rho_{i}^{E}(z ; E)+\rho_{i}^{B}(z ; E)\right] v_{i}+\rho_{i}^{W}(z ; E) l\left(v_{i}\right)-X_{i}(z ; E)
$$

Thus

$$
\frac{\partial X_{i}\left(v_{i} ; E\right)}{\partial v_{i}}=\frac{\partial\left[\rho_{i}^{E}\left(v_{i} ; E\right)+\rho_{i}^{B}\left(v_{i} ; E\right)\right]}{\partial v_{i}} v_{i}+\frac{\partial \rho_{i}^{W}\left(v_{i} ; E\right)}{\partial v_{i}} l\left(v_{i}\right)
$$

which, integrating by parts, implies

$$
\pi_{i}\left(v_{i} ; E\right)=\int_{0}^{v_{i}}\left[\rho_{i}^{E}(z ; E)+\rho_{i}^{B}(z ; E)+l^{\prime}(z) \rho_{i}^{W}(z ; E)\right] d z+\pi_{i}(0 ; E) .
$$

The Lemma follows. QED.

\section{Proof of Proposition 2:}

We can write the expected revenue for the seller as the difference between surplus and expected rents for the buyers. Thus, the sellers revenue are given by

$$
\begin{aligned}
& \sum_{i=1}^{n} \sum_{\theta_{i}=E, W} \frac{1}{2}\left\{\int _ { 0 } ^ { 1 } \left[\left(\rho_{i}^{\theta_{i}}\left(v_{i} ; \theta_{i}\right)+\rho_{i}^{B}\left(v_{i} ; \theta_{i}\right)\right) v_{i}+\rho_{i}^{\overline{\theta_{i}}}\left(v_{i} ; \theta_{i}\right) l\left(v_{i}\right)\right.\right. \\
& \left.\left.-\int_{0}^{v_{i}}\left[\rho_{i}^{\theta_{i}}\left(z ; \theta_{i}\right)+\rho_{i}^{B}\left(z ; \theta_{i}\right)+l^{\prime}(z) \rho_{i}^{\overline{\theta_{i}}}\left(z ; \theta_{i}\right)\right] d z\right] f\left(v_{i}\right) d v_{i}-\pi_{i}\left(0 ; \theta_{i}\right)\right\},
\end{aligned}
$$

where $\overline{\theta_{i}}$ represents the location type opposite to $\theta_{i}$. Inverting the order of integration in the double integral, the above expression can be written as

$\sum_{i=1}^{n} \sum_{\theta_{i}=E, W} \frac{1}{2}\left\{\int_{0}^{1}\left[\left(\rho_{i}^{\theta_{i}}\left(v_{i} ; \theta_{i}\right)+\rho_{i}^{B}\left(v_{i} ; \theta_{i}\right)\right) J\left(v_{i}\right)+\rho_{i}^{\overline{\theta_{i}}}\left(v_{i} ; \theta_{i}\right) J_{l}\left(v_{i}\right)\right] f\left(v_{i}\right) d v_{i}-\pi_{i}\left(0 ; \theta_{i}\right)\right\}$.

Under the constraint that $\sum_{i=1}^{n} p_{i}^{\theta_{i}}=1$ is always satisfied (that is, that the objects are sold with probability 1 ), and recalling the definition of $\rho_{i}^{\theta_{i}}$ in terms of $p_{i}^{\theta_{i}}$, this is maximized as stated in the text of the proposition. QED. 


\section{Proof of Proposition 3:}

First we compute equilibrium strategies for the right to choose auction when the choice of the winner in the first round is not revealed. We conjecture that the bidding behavior of the first round reveals no information about the location type of the winner. In the second round, bidders bid their willingness to pay for an object the is of type $E$ with probability $1 / 2$ and of type $W$ with probability $1 / 2$. That is, a bidder with valuation type $v$ bids $\frac{v+l(v)}{2}$. In the first round, we compute the equilibrium bidding behavior using the same inferences as we used in the proof of Proposition 1. Thus, if no more than $n-2$ other bidders have dropped (i.e., if there are still 2 or more other bidders left), a bidder expects no profit in the second round of the auction. Thus, she stays up to $P=v$. If only 1 other bidder is left in the auction, a bidder computes what she expects staying and what she expects dropping under the conjecture that the other bidder still active has her same valuation $v$. If the last bidder to drop so far has done so at a price $v_{3}$, dropping means expected rents of $\frac{v+l(v)-\left(v_{3}+l\left(v_{3}\right)\right)}{2}$. As before, staying means expected rents of $v-P$. Thus, the dropping price in the first round would be

$$
P_{1}\left(v_{i} ; v_{n}, v_{n-1}, \ldots, v_{3}\right)=\frac{v_{i}-l\left(v_{i}\right)+\left(v_{3}+l\left(v_{3}\right)\right)}{2} .
$$

Again, this is a strictly increasing function with $P_{1}\left(v_{3} ; v_{n}, v_{n-1}, \ldots, v_{3}\right)=v_{3}$, independent of the location type of the bidder. Thus, we have obtained indeed an equilibrium. In this equilibrium, buyer 1 wins the right to choose in first place, and buyer 2 obtains the object left. The price in the first round is $\frac{v_{2}-l\left(v_{2}\right)+\left(v_{3}+l\left(v_{3}\right)\right)}{2}$, and the price in the second period is $\frac{v_{3}+l\left(v_{3}\right)}{2}$. We can compare this with the expected prices in the right to choose with observability. For instance, for $n=3$, these are respectively

$$
\frac{2 v_{2}+2 v_{3}+2 l\left(v_{3}\right)-\left(l\left(v_{2}\right)+\max \left\{l\left(v_{2}\right), v_{3}\right\}\right)}{4},
$$


and

$$
\frac{v_{3}+2 l\left(v_{3}\right)+\min \left\{l\left(v_{2}\right), v_{3}\right\}}{4} .
$$

Thus, announcing the choice of the winner in the first round reduces the expected (with respect to the location type) revenue of the seller by $\max \left\{\frac{v_{3}-l\left(v_{2}\right)}{2}, 0\right\}$.

For general $n$, the expected price in the first period is given by $P_{2}\left(v_{2} ; v_{n}, \ldots, v_{3}\right)$ as defined in the proof of Proposition 1, and the expected price in period 2 is $(k-2)\left(\frac{1}{2}\right)^{k-1} l\left(v_{2}\right)+\left(\frac{1}{2}\right)^{k^{\prime}-2} l\left(v_{3}\right)+(m-2) \sum_{m=3}^{k}\left(\frac{1}{2}\right)^{m-1} v_{m}+\sum_{m=k+1}^{k^{\prime}}\left(\frac{1}{2}\right)^{m-2} v_{m}$. Thus, computing the difference between expected prices in the right to choose without observability and the expected price in the efficient auction, we obtain (for $n>3$ ),

$$
\begin{aligned}
& \left(\frac{1}{2}\right) \max \left\{v_{3}-l\left(v_{2}\right)\right\}+\left(\frac{1}{2}\right)^{3} \max \left\{v_{4}-l\left(v_{2}\right)\right\}- \\
& \sum_{m=6}^{n}(m-5)\left(\frac{1}{2}\right)^{m-1} \max \left\{v_{m}-l\left(v_{2}\right)\right\}-\sum_{m=4}^{n}\left(\frac{1}{2}\right)^{m-2} \max \left\{v_{m}-l\left(v_{3}\right)\right\},
\end{aligned}
$$

which is positive since $\max \left\{v_{3}-l\left(v_{2}\right)\right\}>\max \left\{v_{m}-l\left(v_{3}\right)\right\}$ for $m>3$, and $\max \left\{v_{4}-l\left(v_{2}\right)\right\}>\max \left\{v_{m}-l\left(v_{2}\right)\right\}$ for $m>4$, and $\sum_{m=6}^{n}(m-5)\left(\frac{1}{2}\right)^{m-1}<\left(\frac{1}{2}\right)^{3}$, whereas $\sum_{m=4}^{n}\left(\frac{1}{2}\right)^{m-2}<\left(\frac{1}{2}\right)$. QED. 